\title{
What are the Current Advances Regarding Iron Oxide Nanoparticles for Nanomedicine?
}

\section{Laurence Motte*}

Department of Physical Chemistry, Université Paris, France

Nanomaterials have a significant potential application in the fields of physics, chemistry, biology, medicine and material science because of their unique electronic, optical, thermal and catalytic properties. It has also been generally acknowledged that the physicochemical properties of nanomaterials strongly depend on their shape and size besides their inherent chemical constitutions. To improve the dispersibility of nanoparticles in suitable solvents and to translate intrinsic properties of nanoparticles to these extended applications, the nanoparticle surface functionalization itself is a key factor.

An outstanding class of functional materials with potential applications in almost all fields of technology consists of metal oxide nanoparticles and especially iron oxide $(\mathrm{FeOx})$ magnetic nanoparticles. Iron oxide nanocrystals have been of much scientific and technological interest over the past decades, in particular for various magnetic applications, such as in magnetic recording devices, ferrofluids, magnetic refrigeration systems, and catalysis. Due to the intrinsic multifunctionality of MNPs, the recent focus is in Nanomedicine.

Nanomedecine is an emerging interdisciplinary research field related to the use of hybrid nanoparticles in life sciences, especially for improvement of diagnosis, prevention and treatment of disease. Within the different chemical compositions of nanomaterials, superparamagnetic iron oxide nanoparticles (SPIONs) are certainly the most promising material for medical applications. The most commonly utilized forms of magnetic nanoparticles are the iron oxide $\gamma-\mathrm{Fe}_{2} \mathrm{O}_{3}$ (maghemite) and $\mathrm{Fe}_{3} \mathrm{O}_{4}$ (magnetite). Below approximatively $30 \mathrm{~nm}$ in size, such particle exhibit superparamagnetism. Briefly, the nanoparticles consist of single crystal domain and thus present a single magnetic moment. With the help of thermal energy, however, the magnetic moment can overcome the anisotropy barrier and spontaneously flip from one direction of anisotropy to another thus, the net magnetization of a particle averages to zero. A collection of magnetic nanoparticles consequently displays negligible magnetic remanence. When placed inside an external magnetic field, the magnetic moments align in the direction of the field lines and yielding a fixed magnetization direction. The intrinsic interaction of magnetic nanoparticle with applied magnetic field gradients makes these particles attractive for a large panel of biomedical applications such as immunoassays, imaging, nanovehicles and drug carriers or hyperthermia.

Immunoassay is one of the most important methods in the field of clinical and environmental analysis. Magnetic nanoparticles as new labels in immunoassays have received considerable attention because they are inexpensive to produce, physically and chemically stable and biocompatible. In addition, biological samples exhibit virtually no magnetic background, and thus highly sensitive measurements can be performed in turbid or visually obscured samples without further processing, by detection of magnetic signal $[1,2]$.

Magnetic Resonance Imaging (MRI) is a very powerful imaging tool due to its non-invasive nature, high spatial resolution and tomographic capabilities. The applications of MRI using SPION contrast agents have steadily increased over the past decade, offering the advantage of high spatial resolution of contrast differences between malignant and healthy tissues. Indeed SPIONs are excellent MRI contrast agents because of their transverse relaxation $\mathrm{T}_{2}^{*}$ property, as compared to other MRI agents such gadolinium-chelate contrast agents. These $\mathrm{T}_{2}$ relaxation effects result in a signal reduction on $\mathrm{T}_{2}$-weighted images, thus providing a dark or negative contrast due to the induction of strong magnetic susceptibility on the water protons around the particles. SPIONs are currently used to detect liver lesions since these particles are taken up by macrophages. SPIONs are also attractive as sensitive contrast agents for cancer imaging.

For drug delivery, most of nanoparticles used are hollow structures such as liposomes. This approach has often resulted in improved pharmacokinetics and pharmacodynamics of the drugs, translating into an improved pharmacological efficacy and safety. However the selective and targeted release of the drug out of the liposome is not easy to achieve. SPIONs can be also used for drug delivery coating the nanoparticle surface with therapeutic molecules. Hence, this hybrid nanosystem combines both MRI (diagnostic) and therapeutic applications. This strategy is referred to theranostic strategy $[3,4]$. For example, iron oxide nanoparticles were surface passivated with a clinically relevant antitumour agent: alendronate. These hybrid nanoparticles present anti-cancer properties. In vivo experiments confirmed the therapeutic efficacy of these hybrid nanoparticles in presence of a magnetic field [4]. A combination of both properties (magnetic targeting and liposomal drug encapsulation) was also evaluated on various human cancer cell lines [5]. An effective 100\% cytotoxic effect was achieved with alendronate grafted-SPIONs entrapped into liposomes and exposed to a magnetic field.

Hyperthermia is used for cancer treatment, in order to kill cancer cells by induction of a temperature elevation in body including local, regional, and whole-body. Since SPIONs are excitable within magnetic field these particles present a specific interest to deliver local heating (hyperthermia).

Since the magnetic nanoparticle behavior strongly depend on size, chemical composition, morphology, surface chemistry and state of aggregation of the particles, preparation methods to produce nanoparticles with unique properties are required. Many approaches

*Corresponding author: Laurence Motte, Department of Physical Chemistry, Université Paris, France, Tel: 330-148-387-707; Fax: 330-148-387-625; E-mail: laurence.motte@univ-paris13.fr

Received November 20, 2012; Accepted December 10, 2012; Published December 17, 2012

Citation: Motte L (2012) What are the Current Advances Regarding Iron Oxide Nanoparticles for Nanomedicine? J Bioanal Biomed 4: e110. doi:10.4172/1948 593X.1000e110

Copyright: (c) 2012 Motte L. This is an open-access article distributed under the terms of the Creative Commons Attribution License, which permits unrestricted use, distribution, and reproduction in any medium, provided the original author and source are credited. 
Citation: Motte L (2012) What are the Current Advances Regarding Iron Oxide Nanoparticles for Nanomedicine? J Bioanal Biomed 4: e110. doi:10.4172/1948-593X.1000e110

Page 2 of 2

have been employed to prepare iron oxides nanoparticles, including: co-precipitation, sol-gel methods, thermal decomposition, micro emulsion and hydrothermal methods. In comparison to these wellestablished approaches, the use of microwave irradiation represents a relatively new strategy in nanoparticle synthesis and surface functionalization [6-8].

\section{References}

1. Motte L, Benyettou F, de Beaucorps C, Lecouvey M, Milosevic I, et al (2011) Multimodal superparamagnetic nanoplatform for clinical applications: immunoassays, imaging \& therapy. Faraday Discuss 149: 211-225.

2. de Montferrand C, Lalatonne Y, Bonnin D, Motte L, Monod P (2012) Non linear magnetic behavior around zero field of an assembly of superparamagnetic nanoparticles. Analyst 137: 2304-2308.

3. Lalatonne Y, Paris C, Serfatty JM, Weinmann P, Lecouvey M, et al. (2008)
Bis-phosphonates-ultra small superparamagnetic iron oxide nanoparticles: a platform towards diagnosis and therapy. Chem Comm 2553-2555.

4. Benyettou F, Lalatonne Y, Chebbi I, Di Benedetto M, Serfaty JM, et al. (2011) A multimodal magnetic resonance imaging nanoplatform for cancer theranostics. Phys Chem Chem Phys 12: 2735-2740.

5. Benyettou F, Chebbi I, Motte L, Seksek O (2011) Magnetoliposome for Alendronate Delivery. J Mater Chem 21: 4813-4820.

6. Benyettou F, Guenin E, Lalatonne Y, Motte L (2011) Microwave Assisted Nanoparticle Surface Functionalization. Nanotechnology 22: 55-102.

7. Milosevic I, Jouni H, David C, Warmont F, Bonnin D, et al. (2011) Facile Microwave Process in Water for the Fabrication of Magnetic Nanorods. J Phys Chem C 115: 18999-19004.

8. Guénin E, Hardouin J, Lalatonne Y, Motte L (2012) Bivalent alkynebisphosphonate as clickable and solid anchor to elaborate multifunctional iron oxide nanoparticles with microwave enhancement. J Nanopart Res 14: 965. 